\title{
A Detection Model of Granary Storage Quantity Based on Deep Learning
}

\author{
Xin Zhang ${ }^{1, a}$, Dexian Zhang ${ }^{2, \text { b, }}$ \\ ${ }^{1}$ School of Information Science and Engineering, Henan University of Technology, Zhengzhou, \\ China \\ ${ }^{2}$ Grain Information Processing and Control, Key Laboratory of Ministry of Education, Zhengzhou, \\ China \\ ayimachengxin@163.com,bzdx@haut.edu.cn
}

Keywords: Storage Quantity Monitoring; Pressure Sensor; Deep Learning; Advantage.

\begin{abstract}
According to the special significance of grain and all the detection methods of granary storage quantity, this paper select detection methods of granary storage quantity based on pressure sensors figured with high universality, practicability and reliability, analyze them and put forward a improved version of detection method based on deep learning. This paper then analyze its feasibility and introduce its implementation method and finally summarize the advantage of deep learning in detection methods of granary storage quantity.
\end{abstract}

\section{Introduction}

With the development of agricultural technologies, our country's grain output has been steadily increasing, and the detection methods of granary storage quantity are also updating. At present, most of the grain reserve depots in our country has been equipped with monitoring system. The modern granary detection technology helps reinforce the regulation of grain reserve depots and detect the granary storage quantity precisely, instantly and efficiently. Ren Gongchang and Zhang Ge give detailed review and comparison on the development of granary detection methods (Progress and Prospect of Grain Deport Measurement Techniques)and reached to an conclusion that the detection method based on pressure sensors brought up by Zhang Dexian is highly universal, practical and reliable.

\section{A Detection Method of Granary Storage Quantity Based on Pressure Sensor}

Thedetection method research of granary storage quantity based on pressure sensor focus on first the relations between granary grain weight and grain pressure distribution, second the generatioin machnism of grain bottom pressure and wall pressure and their interaction effect, third granary storage quantity detection method based on the value of the best detection position and its modelingapproach. Then this paper gives a theoretical model of detection, a detection model based on bottom pressure mean estimation(Granary storage quantity detection method based on bottom pressure estimation), a detection model based on bottom and wall pressure mean estimation, a detection model based on SVR(On-line granary storage weight detection based on SVR).

\subsection{Theoretical model}

There are flat granaries, squat silos, and common silos. When filling in the grain, the top of the grain must bu leveled, the grain in the flat granaries presents as cubes while the grain in the squat silos, and common silos presents as cylinders. Through pressure analysis of the grain,we can conclude the

$$
W=\int_{S_{B}} Q_{B}(s) d s+\int_{S_{F}} f_{F}(s) Q_{F}(s) d s
$$

In this formula, W repreents grain weight; $S_{B}, S_{F}$ represents the bottom and wall of the grain; $Q_{B}(s), Q_{F}(s)$ represents the pressure of $s$ point on the grain bottom and wall. $f_{F}(s)$ represents the frictionalcoefficient between the grain bottom $S_{B}$ and grain wall $S_{F}$. In the formula (1-1), the first block represents the grain bottom pressure, the second represents the grain wall friction, the addtion 
of those two equals the grain weight. We can indicate that for a specific grain, the grain weight relates only to the $Q_{B}(s)$ distribution, $Q_{F}(s)$ distribution, $f_{F}(s)$, and the shape and size of bottom $S_{B}$ and wall $S_{F}$.

\subsection{Detection Model Based on Bottom Pressure Mean Estimation}

Detection Model Based on Bottom Pressure Mean Estimation means we measure several positions' pressure on the grain bottom, estimate the mean value, and then calculate the grain weight.

$$
\hat{W}=A_{B}\left(a_{0}+a_{1} \bar{Q}_{B L}(s)+a_{2} \bar{Q}_{B L}(s)^{2}\right)
$$

The formula (1-2)is a detection model based on bottom pressure mean estimation.

\subsection{Detection Model Based on Bottom and Wall Pressure Mean Estimation}

Detection model based on bottom and wall pressure mean estimation means that we place pressure sensors in different positions to build model to estimate the value of $\bar{Q}_{B}(s)$ and $\bar{Q}_{F}(s)$, and use the value to compensate for the interaction effect between $\bar{Q}_{F}(s)$ and $\bar{Q}_{B}(s)$ and thus increase the accuracy.

$$
\hat{W}=A_{B}\left(b_{0}+b_{1} \bar{Q}_{B}\left(s_{\text {Inner }}\right)+b_{2} \bar{Q}_{B}\left(s_{\text {Outer }}\right)+b_{3} \bar{Q}_{B}\left(s_{\text {Inner }}\right) \bar{Q}_{B}\left(s_{\text {Outer }}\right)+b_{4} \bar{Q}_{B}\left(s_{\text {Inner }}\right)^{2} \bar{Q}_{B}\left(s_{\text {Outer }}\right)\right)
$$

In this formula, $\bar{Q}_{B}\left(s_{\text {Outer }}\right)$ represents the mean value of the outer sensors, and $\bar{Q}_{B}\left(s_{\text {Inner }}\right)$ represents the mean value of the inner sensors.

$$
\begin{aligned}
\hat{W}= & A_{B}\left(b_{0}+b_{1} \bar{Q}_{B}\left(s_{\text {Inner }}\right)+b_{2} \bar{Q}_{B}\left(s_{\text {Inner }}\right)^{2}\right. \\
& +b_{3} \bar{Q}_{B}\left(s_{\text {Outer }}\right)+b_{4} \bar{Q}_{B}\left(s_{\text {Inner }}\right) \bar{Q}_{B}\left(s_{\text {Outer }}\right)+b_{5} \bar{Q}_{B}\left(s_{\text {Inner }}\right)^{2} \bar{Q}_{B}\left(s_{\text {Outer }}\right) \\
& +b_{6} \bar{Q}_{B}\left(s_{\text {Outer }}\right)^{2}+b_{7} \bar{Q}_{B}\left(s_{\text {Inner }}\right) \bar{Q}_{B}\left(s_{\text {Outer }}\right)^{2}+b_{8} \bar{Q}_{B}\left(s_{\text {Inner }}\right)^{2} \bar{Q}_{B}\left(s_{\text {Outer }}\right)^{2} \\
& \left.+b_{9} \bar{Q}_{B}\left(s_{\text {Outer }}\right)^{3}+b_{10} \bar{Q}_{B}\left(s_{\text {Inner }}\right) \bar{Q}_{B}\left(s_{\text {Outer }}\right)^{3}+b_{11} \bar{Q}_{B}\left(s_{\text {Outer }}\right)^{4}\right)
\end{aligned}
$$

$\mathrm{b}_{0}$ to $\mathrm{b}_{11}$ in this formula are the regressioncoefficients of the model.

$$
\begin{aligned}
\hat{W}= & A_{B}\left(b_{0}+b_{1} \bar{Q}_{B}\left(s_{\text {Inner }}\right)+b_{2} \bar{Q}_{B}\left(s_{\text {Inner }}\right)^{2}+b_{3} \bar{Q}_{B}\left(s_{\text {Inner }}\right)^{3}+b_{4} \bar{Q}_{B}\left(s_{\text {Inner }}\right)^{4}\right. \\
& +b_{5} \bar{Q}_{B}\left(s_{\text {Outer }}\right)+b_{6} \bar{Q}_{B}\left(s_{\text {Inner }}\right) \bar{Q}_{B}\left(s_{\text {Outer }}\right)+b_{7} \bar{Q}_{B}\left(s_{\text {Inner }}\right)^{2} \bar{Q}_{B}\left(s_{\text {Outer }}\right) \\
& +b_{8} \bar{Q}_{B}\left(s_{\text {Outer }}\right)^{2}+b_{9} \bar{Q}_{B}\left(s_{\text {Inner }}\right) \bar{Q}_{B}\left(s_{\text {Outer }}\right)^{2}+b_{10} \bar{Q}_{B}\left(s_{\text {Inner }}\right)^{2} \bar{Q}_{B}\left(s_{\text {Outer }}\right)^{2} \\
& \left.+b_{11} \bar{Q}_{B}\left(s_{\text {Outer }}\right)^{3}+b_{12} \bar{Q}_{B}\left(s_{\text {Inner }}\right) \bar{Q}_{B}\left(s_{\text {Outer }}\right)^{3}+b_{13} \bar{Q}_{B}\left(s_{\text {Outer }}\right)^{4}\right)
\end{aligned}
$$

Formula (1-3),(1-4)and (1-5)is the detection model based on bottom and wall pressure mean estimation

\subsection{Detection Model Based on SVR}

\subsubsection{SVR Detection Model Based on Sensor OutputSequence}

For specific granary and grain sample $S$, we regulate the value of $W_{m} / A_{B}^{m}$ and $Q_{B L}^{m}$ to [-1,1], and then can build the granary weight detection model based on SVR

$$
\hat{W}=A_{B}\left(\sum_{j=1}^{l} \alpha_{j} K\left(Q_{B L}, Q_{B L}^{j}\right)+b\right)
$$

In this formula, $K\left(Q_{B L}, Q_{B L}^{j}\right)$ is kernel, $\alpha_{j}, b$ is parameter gained by SVM, $\alpha_{j} \neq 0 ; Q_{B L}^{j}$ is the related supportvectorpoint, $j=1, \ldots, l$.

\subsubsection{SVR Model Based on Mean Value of Pressure Sensor}

For specific granary and grain sample $S$, we regulate the value of $W_{m} / A_{B}^{m}$ and $Q_{B M}^{m}$ to [-1,1], and then can build the granary weight detection modeL:

$$
\hat{W}=A_{B}\left(\sum_{j=1}^{l} \beta_{j} \exp \left(-\gamma\left\|Q_{B L}-Q_{B L}^{j}\right\|^{2}\right)+b\right)
$$

In this formula, $\beta_{j}, b$ is parameter gained by $\mathrm{SVM}, \beta_{j} \neq 0 ; Q_{B M}^{j}$ is the related supportvectorpoint, $j=1, \ldots, l$.

Figure 1 is the detection model diagram based on mean value of pressure sensor and SVR 


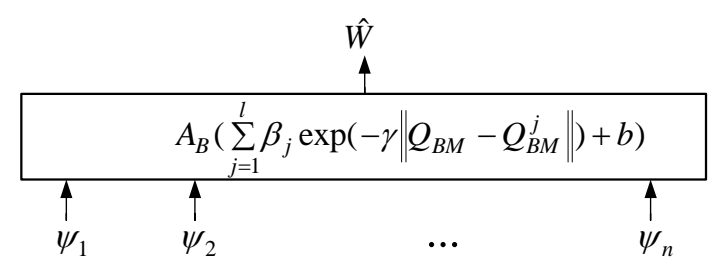

Figure 1: Detection Model Diagram

Formula(1-7)is the detection model diagram based on mean value of pressure sensor and SVR. Compared with formula(1-6), this model can decrease the need of compatibility among sensors but will also dcrease the detection accuracy and need more pressure sensors.

\section{Feasibility Analysis on Building Models for Detection Method of Granary Storage Quantity Based on Deep Learning}

Theoretical model is applicable to large granaries, but not to small granaries; detection model based on bottom pressure mean estimation is only applicable to granaries with small CB/ABand for low accuray; detection model based on bottom and wall pressure mean estimation is applicable to flat granaries, squat silos, and need more pressure sensors; detection model based on sensor output sequence SVR is more complicated, need more models and calculation andrequires mucn on sensor compatibility ; detection model based on sensor mean value SVR requires less on sensor compatibility, but will decrease the detection accuracy and need more sensors. In the light of this background, a more stable applicable and accurate detection model is needed.

Deep learning is brought up in 2006, and ever since then, it has greatly changed algorithmdesign in so many fields,such as voicerecognition,imageclassification and text comprehension, and generate a new model that starts from training data,through end-to-end model and then reaches the ouput directly. This has not only made the whole process easier, but also improved the accuracy, because it can adjust itself in every step of the mission. With the age of big data coming up and the development of hardwares such as GPU, deep learning is more powerful than ever. It can use all the data, automatically learn astract concepts and change the data into some kind of knowledge. Deep learning is introduced into machine learning to reach its original purpose-artifical intelligence. Deep learning is the inner form of data sample learning, its ultimate goal is learn like a human, with analyzing and learning ability. Deep learning, in its very nature is to build a multiple-level machine learning model, which trains by enormous data and a lot of representative information and then classifies and predicts on the sample, improve the accuracy. This process uses deep learning model to reach the goal of feature learning. Deep learning model is different from traditional shallow learning model in: 1 . deep learning model has more levels, usually more than 5 ,sometimes can reach 10 or even more; 2. emphasize on the importance of feature learning, that is, to extract information level by level and change the original feature space to another feature space, and thus makes the classification or prediction easier.

The detection methodof granary storage quantity based on pressure sensors is to build model on the data collected from pressure sensors. The data increases with time and provides enough training samples for deep learning to build model. With enormous datasets, deep learning can automatically extract feature and build the fittest model, and then find the features and relations among the datasets. This method has greatly minimize the deviation when building models.

\section{Analysis on Implementation}

The basic logic of deep learning: assume there is system $\mathrm{S}$ and it has $\mathrm{N}$ levels(S1, . , Sn), the input

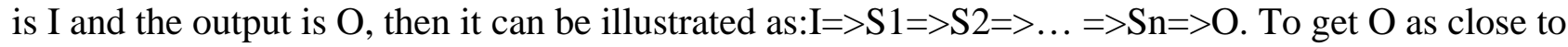
I as possible, we can adjust the parameter in the system, then we will get a series of features of I: S1, $\mathrm{S} 2, \ldots, \mathrm{Sn}$. Among the levels, the output of one level will be the input of another level to achive the level-by-level expression of the input data. 
We can build frame by Caffe, and perform sample training with the enormous data collected from pressure sensors, and then get the parameter and thedetection model of granary storage quantity.

Training process:(1) bottom-up unsupervised learning: use the non-label data to train every level's parameter, this is a unsupervised training process(also a feature learning process), where it differs most from the traditionalneuralnetwork. To be exact, train thefirst level with non-label data and get the parameter of the first level, and proceed similarly to the $\mathrm{N}$ level. This is called network pretraining.

(2)Top down supervised learning: after the pre-training, use the labeled data to do network discriminative trainning, during which the deviation tranfers from top down. Pre-training is like the random initialization of the traditioanl neural network. But the first step of deep learning is gained by non-label data training, its initial value is near global optimum. So the good effect of deep learning is mostly due to its first step of feature learning.

\section{Expected effect and future exploration}

Detection models of granary storage quantity based on deep learning can get closer to ultimate detection goal_— artificial intelligent detection. The detection models and methods at present need manual work to find the feature and pattern of data, which is sometimes influeced by the types of granary or the grain. The usage of deep learning in detection methods of granary storage quantity can minimize this influence. The granary will need only to be filled in normally, the detection system can do all the work by deep learning and get the result of the granary storage quantity. Compared with manual feature extraction method, the deep learning get more representative data features. Therefore, detection models of granary storage quantity based on deep learning if more applicable to the modernization need and the future development.

\section{References}

[1]. Ren Gong-chang,Zhang Ge,Liu Peng.Progress and Prospect of Grain Depot Measurement Techniques[J]. The Food Industry,2017(2):241-244.

[2]. Zhang De-xian, Yang Tie-jun, Fu Hong-liang, Fan Chao, Zhang Yuan. An Online Detection Model of Granary Storage Quantity[J].Acta Automatica Sinica,2014,40(10):2213-2220.

[3]. Zhang De-xian,Yang Tie-jun,Fu Hong-liang,Fan Chao,Zhang Yuan.An On-Line Detection Method of Granary Storage Quantity Based on Pressure Sensor[J].Journal of the Chinese Cereals and Oils Association,2014,29(4):98-103.

[4]. Zhang De-xian,Zhang Miao,Zhang Qing-hui,Zhang Yuan.Granary storage quantity detection method based on bottom pressure estimation[J].Transactions of the Chinese Society of Agricultural Engineering,2017(5):287-294.

[5]. Wu An-cheng.Neural network and Deeo learning[M].Beijing:Eletronic Industry Press,2016. 